Horizons philosophiques

\title{
La contribution de Stephen Clark à la philosophie sur Internet
}

\section{Stephen Clark}

Volume 6, numéro 2, printemps 1996

La philosophie sur Internet

URI : https://id.erudit.org/iderudit/801015ar

DOI : https://doi.org/10.7202/801015ar

Aller au sommaire du numéro

\section{Éditeur(s)}

Collège Édouard-Montpetit

\section{ISSN}

1181-9227 (imprimé)

1920-2954 (numérique)

Découvrir la revue

\section{Citer cet article}

Clark, S. (1996). La contribution de Stephen Clark à la philosophie sur Internet. Horizons philosophiques, 6(2), 95-109. https://doi.org/10.7202/801015ar d'utilisation que vous pouvez consulter en ligne.

https://apropos.erudit.org/fr/usagers/politique-dutilisation/ 


\section{LA CONTRIBUTION \\ DE STEPHEN CLARK \\ À LA PHILOSOPHIE SUR \\ INTERNET}

\section{Introduction}

Entouré de son équipe, Stephen Clark dresse la carte de la philosophie sur l'Internet. Les résultats ont de quoi étonner : après avoir parcouru son survol et exploré les profondeurs inouïes de sa page WWW, on pourrait croire qu'il y a plus de philosophie dans la réalité virtuelle qu'il n'y en a, en réalité, dans les cegeps, les universités ou sur la place publique. Quoi qu'il en soit, les ressources sontmultiples, des Gophers aux journaux électroniques en passant par les groupes de discussion, les babillards électroniques etj'en passe, la philosophie estprésente sur tous les fronts. Reste à voir si toute cette activité est philosophiquement intéressante. II faut savoir que dans -l'hyperespace, on se conduit de manière plus cavalière qu'on a coutume de le faire dans la vie réelle. Ainsi, sur la page de philosophie de Sean Cearly, se trouve en bonne place un lien... à son casier judiciaire. Selon lui, «il ne faut jamais se fier à un philosophe qui n'a pas été arrêté».

C'est peut-être au sens de Wittgenstein qu'il faut prendre cet arrêt ("on doit pouvoir arrêter de philosopher à volonté»), lui qui a aussi sa page sur le WWW, agrémentée d'images de photographies connues, dont une où on a ajouté une auréole. Pour ceux qui ont un écran couleurs et un accès à Mosaic ou une copie du programme Slipknot (qui fonctionne sur Windows et donne pour trente dollars au lieu de trente dollars par mois la possibilité de récupérer des images), l'effet est saisissant. Les liens et les documents afférents sont intéressants, bien qu'il faille s'attendre à trouver le domaine de la philosophie plus vaste et touffu qu'on ne le définirait dans les départements de philosophie orthodoxes (à noter qu'à Diversity University, on dispense des cours «en ligne»). Les «nouveaux philosophes» 
sont Ayn Rand, Leonard Peikoff, etc., et malgré le mépris que peuvent manifester à leur égard certains professeurs d'Oxford, les uns et les autres ont leur place dans le réseau des réseaux. Ce qui nous laisse avec une définition de la philosophie plus large qui est, comme Stephen Clark semble le dire dans son Guide de ressources, dans le pire des cas celle d'hommes et de femmes relativement jeunes, ayant passé par l'université.

\section{Une vision d'ensemble}

(Traduction de «Stephen Overview», le premier élément du site «Philosophy at large» de Stephen Clark)

\section{La petite histoire}

II y avait peu de philosophes jouissant d'un accès sur le WWW dans les années 89. Les humanités avaient l'Humanist, une création de Willard McCarty, de l'Université de Toronto. Au début, cette liste était occupée par les annonces diverses et les querelles académiques. Celles-ciattiraientparfois passablement l'attention, touten étant prudemmentmodérées par le fondateur. Les choses furent beaucoup plus tranquilles pendant la période où la liste fut déplacée à l'Université Brown. Et maintenant que Willard a repris les rênes, les choses peuvent encore changer. Ailleurs au Canada, Nollaig Mackenzie a lancé Philosop à l'Université York, ce qui lui a permis de servir la communauté philosophique nord-américaine. Or la grande force de Philosop, depuis le début, ce sont ses archives; les annonces et les textes sont emmagasinés et peuvent ultérieurement être récupérés et commentés. Le but a toujours été de permettre des échanges rapides d'informations, et les membres n'ont jamais été très accommodants sur la discussion publique de problèmes philosophiques. La liste est dernièrement passée entre les mains d'Istvan Berkeley (Université de l'Alberta). Barry Floyd avait cependant tenté de répondre au besoin de discussion publique en initiant NSP-L (Noble Savage Philosophers') à I'Université Brown et sa liste fonctionna bien de concert avec Philosop jusqu'en 94, lorsque Barry dut l'abandonner pour d'autres occupations. Le bavardage philosophique se déplaça alors vers d'autres listes. 
C'est aussi en 94 que je réussis à persuader les services informatiques de l'Université de Liverpool que nous pourrions avoir la chance d'établir une liste valable pour l'ensemble du Royaume-Uni. PHILOS-L fut d'abord créée à partir de mon répertoire personnel, et le mot fut donné oralement ou par courrier électronique. Depuis lors, la liste s'est étendue à travers le monde, bien qu'elle reste dominée par le monde anglophone et centrée sur les îles Britanniques. Avec PHILOSOP et APHIL-L (Australie) elle fait partie de la triade des listes philosophiques générales. Comme les deux autres listes, elle sert essentiellement à l'échange d'informations, tout en étant plus accueillante aux débats philosophiques occasionnels. Selon le vœu de son fondateur, elle suit la règle louable d'une anarchie tempérée par le despotisme.

Outre les trois listes d'intérêt général PHILOSOP, PHILOS$L$ et Aphil-L, il existe également une foule innombrable d'associations, qui comprennent les groupes du réseau Usenet, les listes dotées ou non d'un modérateur, les MOO, les Gopher et sites WWW. Je commençai à les dénombrer à partir des annonces faites sur NEW-LIST@ vm1.nodak.edu, ou des listes académiques produites par Diane Kovacs. Je fournissais mon rapport sur demande, et le mettais à jour quand je trouvais le temps nécessaire. Le projet de l'Université du Michigan devait propulser ce rapport sur la scène internationnale. Peter Morville produisit une liste plus complète et plus soignée, en partie à partir de mes recherches. Je suis responsable de la révision de cette liste que je tente de garder à jour et si elle fait toujours partie du projet Clearinghouse de l'Université du Michigan, les différentes versions successives sont maintenant rassemblées à Liverpool. Arno Wouters, dans les Pays-Bas, a créé à la même époque un site Gopher très intéressant. Pour une description, cliquez ici (lorsque vous êtes en ligne). Finalement, à Monash, Dey Alexander construisit à partir de ces deux ressources et de ses propres recherches une autre liste de listes, «Philosophy in Cyberspace", qui a depuis été produite en hypertexte par le département de Philosophie de Bristol. Parmi toutes celles qui existent actuellement, je retiens surtout le groupe de listes 
dirigées par Kent Palmer (Dialognet) et Lance Fletcher (Freelance Academy). L'essai de Fletcher sur le «slow reading" fournit des conseils judicieux sur la manière, justement, de lire les textes lentement et avec respect.

\section{Perspectives d'avenir}

Heureusement ou malheureusement, les temps continuent de changer. Des listes prospères sont amenées à fermer ou sont déplacées, et on peut facilement manquer l'annonce de ces changements. Plus les Gophers et les pages WWW deviennent courants et plus le problème s'exacerbe. Les individus développent leurs sites mais oublient de les garder à jour. Après un certain temps, de tels sites ne sont plus visités par les internautes expérimentés mais ils restent là, occasionnant du trafic et des plaintes lorsque les liens qu'ils annoncent ne sont plus opérants. N'oublions pas que la plus grande part de ces sites sont maintenus par des bénévoles. II est intéressant pour un individu de construire lui-même ses pages WWW, mais cette tâche prend quand même du temps. D'où il suit que le matériel présenté est le meilleur que je puisse offrir actuellement : s'il comporte des erreurs, vous devriez m'en faire part, mais évitez surtout les pensées revanchardes.

Les pages WWW, comme les menus Gophers, peuvent vous rendre complètement fou: on trouve des collections désorganisées d'éléments parfois intéressants, avec des titres non révélateurs. Personne n'a envie de se brancher pour récupérer un document sur la "philosophie», qui s'avère n'être qu'un rapport d'étudiant sur de malheureux lecteurs du MidWest. Ce dont nous avons besoin c'est d'un peu d'ordre, ou plutôt de plusieurs degrés d'ordre. $\mathrm{Si}$, comme je le pense, il n'y a pas qu'une seule manière d'ordonner la philosophie, il faudra combiner plusieurs manières de faire. Et même s'il n'y a, après tout, qu'une seule bonne méthode, on aura plus de chances de la découvrir à partir des méthodes évolutionnistes. Laissons donc s'épanouir un million de fleurs et voyons lesquelles d'entre elles vont se faner. On remarque que certains organisateurs s'adonnent au prosélytisme; d'autres considèrent que la voie 
royale vers la vérité passe par les taxinomies (ce qui a certainement quelque chose à voir avec leurs différents types de personnalité). Un des efforts les plus intéressants est celui de Principia Cybernetica, en Belgique; un des plus bizarres, à tout le moins pour les goûts humanistes, se retrouve en Australie, avec la page de Mark, qui contient aussi des liens à une masse de littérature anarchiste; Voice of the Shuttle, un site géré par Alan Liu, comprend toutes sortes de liens à des fichiers pertinents pour les humanités, y compris la philosophie.

D'autres pages ne sont en fait que des collections, mais il peut s'agir de collections bien organisées, faites à partir de ce que l'auteur considère comme intéressant à un moment donné. C'est le cas du Guide du cyberpoète sur la culture virtuelle, ou du Café électronique.

Finalement, quiconque désirant construire une page WWW devrait consulter le guide de la NCSA sur le langage HTML. Le Center for Computing in the Humanities de Toronto fournit un lien au dernier livre de lan Graham, intitulé The HTML Sourcebook (John Wiley, 1994). Une fois votre page complétée, si elle a un intérêt pour la philosophie, faites-en l'annonce sur PHILOS-L.

\section{Petit guide de ressources en philosophie sur le net}

(Tiré de «Resources on the Net») 1

On peut rejoindre toutes les ressources énumérées plus bas ainsi que le document précédent à partir de la seule page de «Philosophy at large» de Stephen Clark : «http://www.liv.ac.uk/srlclark/philos.html. On obtient ainsi non seulement des explications mais l'accès aux sites eux-mêmes.

Concernant les humanités, la source électronique actuelle la plus ancienne est la liste Humanist, qui se trouve désormais à nouveau entre les mains de son fondateur, Willard McCarty.

1. La page WWW de Stephen Clark étant régulièrement augmentée et mise à jour, les listes qui suivent sont uniquement représentatives des ressources que l'on peut atteindre à partir de son site. Mais il suffit de taper son adresse pour accéder directement à la dernière version, qui donne directement accès à toutes les ressources énumérées (note de la trad.). 
Les amateurs de philosophie ont accès aux ressources suivantes :

Usenet (groupes); Listserv (groupes); MOO (rencontres virtuelles organisées à l'avance); archives; bibliographies; textes électroniques; journaux électroniques; cours électroniques; pages sociales; description des départements de philosophie à travers le monde; autres sites (Gopher/WWW).

Le Gopher de l'Université de Liverpool présente une longue liste de sites fameux, incluant Yahoo. Suit une liste d'autres sites philosophiques importants :

+ La page de Bjorn

+ La page d'Andrew Burday à l'Université McGill

+ One Stop Philosophy Shop, de Sean Cearley

+ La librairie virtuelle de Dan Brickley

+ La philosophie sur le WWW, d'après Arno Wouter

+ Voice of the Shuttle, d'Alan Liu

+ The Source, par Mary Long

+ La galaxie Einet

+ Le site de Ron Barnette à Valdosta

+ Les pages philosophiques Hopkins de Dennis Des Chene

+ Le Guide de philosophie sur le Net d'Andrew Burday

+ L'Aleph de R. Jeffrey Grace

+ La page de Hong Kong, avec plusieurs liens à des ressources philosophiques

+ La page de philosophie chinoise de Su Tzu

+ Le kit de ressources en philosophie sur Internet de Michael vanPelt

+ La réponse de Provenzano

+ La vaste collection de textes et de liens de Cosma Rohilla Shalizi

+ La page de Joe McCluskey au Raymond Walters College

+ Le site de Peter King à Oxford 


\section{Les groupes du réseau Usenet}

Ces groupes n'ont souvent pas très bonne réputation, étant jugés d'un niveau équivalent aux lignes ouvertes. Certains d'entre eux sont pourtant d'un niveau plus élevé, reflétant, dans le pire des cas, les intérêts d'une classe d'individus qui sont relativement jeunes pour la plupart. La version de Bristol de «Philosophy in cyberspace», de Dey Alexander (entre autres), en dresse la liste.

En philosophie, on retrouve notamment les groupes suivants:

alt.babylon5.uk

alt.individualism

alt.philosophy.Jarf

alt.philosophy.objectivism

comp.ai.philosophy

sci.philosophy.meta

sci.philosophy.tech

sci.psychology.consciousness

sci.psychology.journals.psyche

soc.religion.gnosis

rec.arts.sf.reviews

talk.philosophy.humanism

\section{Les groupes rattachés à un Listserv}

Les listes de listes de Clark/Morville, Dey Alexander et Arno Wouters sont facilement disponibles et régulièrement mises à jour. La page WWW de Dave Horacek présente une liste de listes composite et très bien ordonnée. II existe une foule de listes, incluant celles qu'on trouve à Liverpool : Philos-L; Sophia and Indology; les plus importantes sont Thinknet, Freelance Academy, Spoon Collective et la Bucknell Initiative. Pour la théologie, outre les ressources mentionnées dans le guide Clark/Morville, voir la liste de groupes de discussion par courrier électronique disponible à Durham. Ceux qui voudraient créer 
une liste doivent se référer au Listserv List Owner's Survival Guide.

\section{Les MOO}

II s'agit de rejetons du jeu «Dongeons \& Dragons», c'est-àdire une construction virtuelle où des internautes identifiés par un pseudonyme conversent ou s'adonnentà des jeuxparticuliers. En raison des décalages horaires d'un continent à l'autre, il est difficile de déterminer où et à quel moment des conversations intéressantes se produisent. Ces réunions virtuelles peuvent cependant être annoncées à l'avance, et parfois ça marche. Un des plus anciens est le Lambda-MOO; il y a aussi le PMC-MOO. Toutefois, avant de s'y risquer, il est fortement conseillé de lire "A Rape in Cyberspace» de Julian Dibbell, in The Village Voice, 21 décembre 1993, pp. 36-42. De même, Brad de Long (Berkeley) a produit un bon essai sur les bizarreries de la cyberéalité.

Le colloque Virtual Futures 95, qui s'est tenu à Warwick à la fin de mai, avait établi un camp dans le Lambda-MOO. Gloria McMillan tient une classe virtuelle à Diversity University, où les étudiants peuvent discuter d'un texte qui est projeté dans la pièce. II semble que les discussions portent surtout sur des textes littéraires. Pour voir de quoi il s'agit, faire un telnet en direction de Diversity University (telnet: moo.org:8888). Le login comme visiteur est «co-guest»; taper ensuite «@go \#2673». Finalement, le Café des philosophes se réunit la nuit (une manière de parler) au Mundus Philosophicus, qu'on rejoint également par telnet (comme tous $\mathrm{MOO}$ ). Une autre manière de participerà des assemblées virtuelles, sans avoirà les organiser, est de joindre un des canaux IRC (communication en direct, sans organisation préalable). 


\section{Les archives}

\section{Textes récents}

La plus grosse collection de documents philosophiques se trouve à l'International Preprint Exchange. II s'agit de travaux en cours de production, et les commentaires sont les bienvenus. Ces esquisses sont souvent plus intéressantes que les articles qui finalement paraissent dans les revues spécialisées (même les journaux électroniques). Par ailleurs, certains commencent à intégrer leurs textes à leurs propres pages WWW : s'agit-il de publication? En attendant de pouvoir répondre à la question, on peut consulter :

+ Les pages d'Onar, ainsi que les Principia Cybernetica

+ Les textes de David Chart

+ Ceux de Dave Chalmers sur la conscience, le contenu, l'intelligence artificielle, etc., ainsi que sabibliographie commentée sur la "philosophy of mind"s

+ La réponse de Provenzano

+ Science without Bounds, d'Arthur J. D'Adamo.

+ Stodolsky sur la démocratie en réseau

+ La théorie générale de la religion d'Arlen Wolpert.

\section{Listes archivées}

De nombreuses listes ont maintenant leurs archives, qu'il est possible de consulter ou de récupérer. La liste de listes Clark/Morville contient plusieurs liens à de telles archives.

PHILOS-L et Sophia sont archivées à Liverpool.

\section{Les grands philosophes}

Les grands philosophes sont en train d'acquérir de nouvelles archives. C'est le cas par exemple de :

+ David Hume

+ Friedrich Nietzsche (un site excellent)

+ Charles Sanders Peirce

+Uneautresourcesur Peirce, avec possibilité de récupérer les documents 
+ Martin Heidegger

+ Jacques Maritain

+ Bertrand Russell

+ Ludwig Wittgenstein

+ Leibniz

+ Chomsky

+ Des biographies des grands d'Édimbourg, incluant David Hume et Adam Smith etc.

\section{Suit une liste d'autres projets incluant des documents} archivés :

+ Les archives de l'Economics Working Paper

+ L'esthétique à Louisville : une page créée par l'American Society for Aesthetics et The Journal of Aesthetics and Art Criticism

+ Les Cultural Studies à McGill

+ Le projet Mind

+ Le groupe Philosophy \& Genetics à Chicago

+ Les archives Voegelin à Manchester

+ Le numéro interactif de la revue The Monist, qui sera le volume 80 , à paraître en juillet 97 ; ceux qui ont des suggestions peuvent les envoyer à J. C. Nyiri

+ Origin - Conference of Religions, lié à la liste par courrier électronique Interrel@vm.temple.edu

+ La philosophie kabalarienne, ou comment atteindre la santé mentale, physique et spirituelle

+ L'Institut Ayn Rand, pour une autre manière d'atteindre le but de l'existence

+ Les ressources disponibles sur le XVIIle siècle, à l'Université de Pennsylvanie

+ Souvent, les collections en cours de formation qu'on trouve à partir d'ici prennent la forme du journal électronique. 


\section{Bibliographies, listes d'éditeurs et autres}

\section{Annonces diverses}

+ Humanities Research Board Newsletter

+ THES Gopher SERVICE

+ Éthique de la science : bibliographie et liens

\section{Librairies}

+ Versions électroniques de livres déjà publiés

+ L'Internet Bookshop, des liens aux catalogues de nombreux éditeurs

+ Il existe de nombreuses autres librairies

\section{Éditeurs}

+ Le guide de Routledge sur la philosophie

+ Cambridge University Press

+ Greenwood Press

+ UMI Dissertations Abstracts (en philosophie, religion et théologie)

+ UMI Dissertation Abstracts (autre source)

+ TitleNet

\section{Bibliographies}

+ Informations concernant le Philosopher's Index

+ Sciences sociales

+ La librairie virtuelle des sciences sociales

+ La librairie virtuelle du World-Wide Web (religions)

+ La librairie virtuelle Reference Shelf, incluant un lien à

l'Encyclopedia Britannica Online.

+ ABELL: langue et littérature anglaises

+ Ressources sur le XVIIle siècle

+ Outils de recherche pour bibliophiles

+ La bibliographie de Dave Chalmers sur la Philosophy of Mind

+ Éthique de l'environnement

etc. 


\section{Enseignement}

+ Les nouveaux outils d'apprentissage de J. J. O'Donnell

+ Un choix de cassettes, incluant les géants de la philosophie

+ Quantité de casse-tête logiques

+ Outils favorisant l'apprentissage de la logique

$+C T I$ Textual Studies : programmes logiques

+ Les logiciels logiques de CSLI, incluant le monde de Tarsky

+ Une galerie de portraits

+ Le cours de Steven Palmquist

+ Matériel provenant de l'Université McGill

+ The Internet Education Warehouse

+ La bioéthique à partir de cas d'espèce

+ Liddell-Scott-Jones Oxford Greek Dictionary

+ Informations concernant les vidéos sur Quine et Strawson

\section{Textes électroniques}

Le Gopher de Liverpool en dresse une liste assez complète. La collection de Jason Abbot est encore plus importante, avec des liens en hypertexte. ALEX fournit une importante collection, comme le Center for Electronic Texts in the Humanities de Princeton. Le Guide de Bjorn contient des liens à de nombreuses sources. Voir aussi Internet on a disc.

\section{Pages sociales}

+ American Philosophical Association

+ American Association of Philosophy Teachers

+ Society of Christian Philosophers

+ American Society for Aesthetics

+ The Southern Society for Philosophy and Psychology

+ Un autre site permettant de rejoindre la SSPP

+ Canadian Society for History and Philosophy of Mathematics 
+ Friedrich Nietzsche Society

+ Australian Association of Professional and Applied Ethics

+ British Society for Metaethics

\section{Journaux électroniques}

Voir la liste Clark/Morville ainsi que les annonces de nouveaux journaux. L'hyperjournal de Damien Keown contient des liens à plusieurs journaux électroniques, dont:

+ EJournal (V5N1) - le site donne accès aux textes dans leur intégralité

+ Analysis (Sheffield)

+ International Joumal of Tantric Studies (Italie)

+ Sorites (journal de philosophie en anglais et espagnol)

1. L'International Preprint Exchange, incluant:

+ Ethics (1993-)

+ Journal of the History of Philosophy (1994- )

+ Le sixième congrès de la société leibnizienne internationale (1993-)

+ Philosophy of the Social Sciences (1994-)

+ Poznan Studies in Philosophy of Sciences and Humanities (1990-)

+ Radical Philosophy (1994-)

2. L'Electronic Journal of Analytic Philosophy

3. Psyche Journal of Research on Consciousness

4. Psyche (autre site)

5. LOGBANK - The Polish Electronic Magazine for Logic and Philosophy

6. Postmodern Culture

7. LOGOS

8. Postmodern Jewish Philosophy

9. PSYCOLOQUY

10. ANALYSIS

11. Le numéro interactif de la revue The Monist 
12. Sorites

13. Joumal of Buddhist Ethics

14. Les comptes rendus d'ouvrages de littérature et de philosophie classiques de Bryn Mawr

15. Le Projet Muse

16. Radical Philosophy

17. Electronic Antiquity

18. International Society for Environmental Ethics

19. Arachnion : histoire et littérature anciennes

20. Brown Electronic Article Review Service

21. Electronic Journal of Vedic Studies

22. Chorus : informatique et humanités.

23. Ctheory (anciennement le Canadian Journal of Political and Social Theory)

24. Comptes rendus électroniques : s'adresser à glc@ksuvm.ksu.edu

25. International Journal of Tantric Studies

26. Online Journal of Ethics

27. Classics Ireland

28. La liste de John Labowitz

29. Diotima

30. Villanova Information Law Chronicle

31. IndiaWorld Headlines

\section{Cours électroniques}

1. PhiCyber de Ron Barnette, un cours d'été en philosophie qui se passait «en ligne».

2. Le cours sur Augustin de Jim O'Donnell.

3. Le Chicago Philosophy Project.

4. Un séminaire sur L'Utopie de Thomas More. 
Départements de philosophie à travers le monde

1. La listes des départements de philosophie en Irlande et en Angleterre de McGunigle.

2. Autres départements : voir aussi le Peterson's Education Center.

3. La liste de Bjorn.

4. Département de philosophie de Liverpool.

\section{Autres sites}

La section philosophie (14) de l'arborescence du Gopher BUBL permet de rejoindre de nombreux autres sites. Voir également la liste Clark/Morville.

Stephen Clark (srlclark@liverpool.ac.uk) University of Liverpool, England

Traduction et introduction de Josette Lanteigne 